Šime Malenica

http://dx.doi.org/10.21278/brod70106

\title{
AN ALTERNATIVE SOLUTION FOR INTERACTION OF FLEXURAL GRAVITY WAVES WITH BOTTOM MOUNTED CIRCULAR CYLINDER
}

UDC 530.122.1:534.182

Original scientific paper

\section{Summary}

The problem of interaction of the flexural gravity waves with the bottom mounted circular cylinder is discussed. Both the linear and second order problems are considered and they are solved by using the eigenfunction expansion principles together with the Boundary Integral Equation technique. The linear solution, which was proposed in the litterature by different authors in the past, is explicitely recovered. For the second order problem, a new original solution strategy is proposed and it was shown that, in the case of water waves, a well known semi-analytical solution is also recovered, proving the generality of the proposed approach.

Key words: $\quad$ Flexural gravity waves; Second order; Green's function; Boundary Integral Equations; Eigenfunctions

\section{Introduction}

Interaction of gravity waves with different obstacles received much attention in the past both in the context of water waves $[1,2,3]$ as well as in the context of flexural gravity waves $[4,5,6,7,8,9,10,11,12,13,14]$. Both the linear $[1,6,7,8,11,13]$ and higher order interactions $[2,3,15,16]$ were of concern. The problem of flexural gravity waves is relevant in the context of the ice structure interactions (both for the floating bodies as well as for the cracks which might occur in the ice sheets) and in the context of the elastic floating structures such as floating airports or the elastic plates lying at the water surface. Unlike the problem of water waves where the semi-analytical solution for the vertical circular cylinder, is well mastered and agreed within the community, the solution for the problem of flexural gravity waves is proposed in different forms by the different authors. In the present work an alternative solution is proposed for both the linear and the higher order problems. The potential flow assumptions are adopted and the problem is formulated in frequency domain. The solution methodology relies on the use of the eigenfunction expansion principles either directly or through the definition of the relevant Green's function and the use of the Boundary Integral Equation (BIE) technique. For the case of linear wave scattering, a very similar work was reported in [13] for Cartesian 
geometry and in [6] for circular geometry. The higher order solution is new and was developed based on the same principles as in [3].

\section{Mathematical model}

The basic configuration together with the different boundary conditions for the generic velocity potential $\varphi$ induced by the cylinder, is shown in Figure 1.

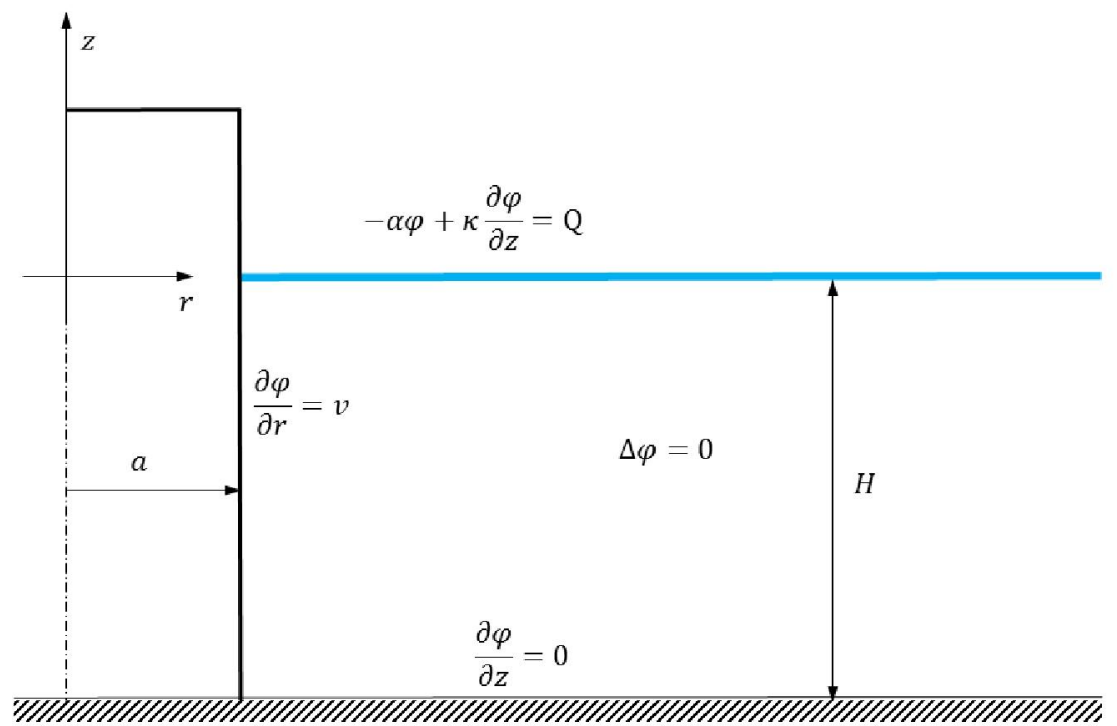

Fig. 1 Basic configuration and definitions

The corresponding boundary value problem (BVP) has the following form:

$$
\left.\begin{array}{ll}
\Delta \varphi=0 & -H \leq z \leq 0 \\
-\alpha \varphi+\kappa \frac{\partial \varphi}{\partial z}=Q(r, \theta) & z=0 \\
\frac{\partial \varphi}{\partial r}=v(a, \theta, z) & r=a \\
\frac{\partial \varphi}{\partial z}=0 & z=-H \\
\varphi \rightarrow 0 & r \rightarrow \infty
\end{array}\right\}
$$

where the operator $\kappa$ is given by the following expression:

$$
\kappa=1-\alpha \frac{M}{\varrho}+\frac{D}{\varrho g} \Delta_{0}^{2}
$$

with $M$ and $D$ being the distributed mass and stiffness of the plate, respectively. 
It is important to mention that, in the case of the flexural gravity waves, the BVP (1) the additional boundary conditions at the plate ends should be specified. At the cylinder, these conditions describe the way in which the plate is attached to the cylinder i.e. clamped, free, ... For example, in the case of the plate clamped to the fixed cylinder the conditions of the zero displacement and zero slope apply:

$$
w(a, \theta)=0 \quad, \quad \frac{\partial w}{\partial r}(a, \theta)=0
$$

The constant $\alpha$ and the functions $v(z, \theta)$ and $Q(r, \theta)$ are specified for each particular problem and here below we define them in the context of the second order wave body interaction theory.

\subsection{Second order theory}

The relevant second order theoretical model for monochromatic flexural gravity waves, was presented in [16] and will not be repeated here in details and we just mention few basic principles. We start by assuming that all the quantities of interest (velocity potential, plate deflection, pressure ...) can be developed into a perturbation series with respect to the small parameter $\varepsilon$ chosen to be the wave steepness:

$$
\varphi=\varepsilon \varphi^{(1)}+\varepsilon^{2} \varphi^{(2)}+o\left(\varepsilon^{2}\right) \quad, \quad w=\varepsilon w^{(1)}+\varepsilon^{2} w^{(2)}+o\left(\varepsilon^{2}\right) \quad, \quad \ldots
$$

This series is introduced into the initial fully nonlinear problem and, at the same time, the Taylor series expansion is used in order to express the different quantities at their instantaneous position as a function of their values at rest. After collecting the terms at different order we obtain:

$O(\varepsilon)$

$$
\alpha^{(1)}=v, \quad \kappa^{(1)}=1-v \frac{M}{\varrho}+\frac{D}{\varrho g} \Delta_{0}^{2}, \quad w^{(1)}=-\frac{1}{i \omega} \frac{\partial \varphi^{(1)}}{\partial z}, \quad Q^{(1)}=0
$$

$O\left(\varepsilon^{2}\right)$

$$
\begin{aligned}
\alpha^{(2)} & =4 v \quad, \quad \kappa^{(2)}=1-4 v \frac{M}{\varrho}+\frac{D}{\varrho g} \Delta_{0}^{2} \\
w^{(2)} & =-\frac{1}{2 i \omega}\left[\frac{\partial \varphi^{(2)}}{\partial z}-\frac{1}{2} \nabla_{0} \varphi^{(1)} \nabla_{0} w^{(1)}+\frac{1}{2} w^{(1)} \frac{\partial^{2} \varphi^{(1)}}{\partial z^{2}}\right] \\
Q^{(2)} & =\frac{1}{2}\left[\kappa^{(2)}\left(\nabla_{0} \varphi^{(1)} \nabla_{0} w^{(1)}\right)-\nabla_{0} \varphi^{(1)} \nabla_{0}\left(\kappa^{(1)} w^{(1)}\right)\right] \\
& +\frac{i \omega}{g} \nabla \varphi^{(1)} \nabla \varphi^{(1)}+\frac{\omega^{2}}{2 g} w^{(1)} \frac{\partial \varphi^{(1)}}{\partial z}-\frac{1}{2} \kappa^{(2)}\left(w^{(1)} \frac{\partial^{2} \varphi^{(1)}}{\partial z^{2}}\right)
\end{aligned}
$$

With respect to the body boundary condition and the quantity $v(, a, z, \theta)$ in (1) we should distinguish two types of problems namely: the radiation and the diffraction. In the case of the radiation the fluid is at rest and the normal velocity $v(a, z, \theta)$ is induced by the prescribed body 
motion, while in the case of the diffraction the body is fixed and the normal velocity is induced by the incident wave. It is however clear that, in both cases, the resulting BVP is of the same form (1) so that exactly the same method can be used for its resolution.

\section{Solution methodology}

As already indicated, the soultion methodology is based on the use of the eigenfunction expansion principles which are employed either directly or through the definition of the corresponding Green's function in a way similar to [13]. In that respect we start by employing the method of separating variables which, for the flow symmetric with respect to $x$ axis $(\theta=0)$, allows writing the solution in the following form:

$$
\varphi(r, \theta, z)=\sum_{m=0}^{\infty} \epsilon_{m} \varphi_{m}(r, z) \cos m \theta
$$

where $\epsilon_{m}=1$ for $m=0$ and $\epsilon_{m}=2$ for $m>0$.

It can also be shown (e.g. [13]) that each Fourier mode $\varphi_{m}(r, z)$ can be further developed into eigenfunction expansion in vertical direction as follows:

$$
\varphi_{m}(r, z)=\sum_{n=-2}^{\infty} f_{n}(z) \varphi_{m n}(r)
$$

where the functions $f_{n}(z)$ denote the eigenfunctions in the vertical direction:

$$
f_{n}(z)=\frac{\cosh \mu_{n}(z+H)}{\cosh \mu_{n} H}
$$

The wave numbers $\mu_{n}$ are roots of the following dispersion equation:

$$
\alpha=\left(\mathcal{M}+\mathcal{D} \mu_{n}^{4}\right) \mu_{n} \tanh \mu_{n} H
$$

where the notation $\mathcal{M}=1-\alpha M / \varrho$ and $\mathcal{D}=D / \varrho g$ was introduced in order to simplify the writing.

The dispersion equation has two real roots $\left( \pm \mu_{0}, \mu_{0}>0\right)$, infinite number of pure imaginary roots $\left( \pm \mu_{n}= \pm i k_{n}, k_{n}>0, n=1, \infty\right)$ and four complex roots $\left(\mu_{-4}=-a_{0}-i b_{0}, \mu_{-3}=a_{0}-i b_{0}\right.$, $\mu_{-2}=-a_{0}+i b_{0}, \mu_{-1}=a_{0}+i b_{0}$ with $\left.a_{0}>0, b_{0}>0\right)$. In the present analysis we follow the procedure from [8] and we restrict ourselves to the roots $\mu_{-2}, \mu_{-1}, \mu_{0}, \mu_{n}=i k_{n}, n=1, \infty$.

It is important to mention that the eigenfunctions $f_{n}(z)$ are not orthogonal in a classical sense but they obey the following orthogonal relation:

$$
\int_{-H}^{0} f_{m}(z) f_{n}(z) d z+\frac{\mathcal{D}}{\alpha}\left(f_{m}^{\prime \prime \prime} f_{n}^{\prime}+f_{m}^{\prime} f_{n}^{\prime \prime \prime}\right)_{z=0}=\frac{\delta_{m n}}{2 \mathcal{C}_{n}}= \begin{cases}0 & n \neq m \\ \frac{1}{2 \mathcal{C}_{n}} & n=m\end{cases}
$$

The Green's function corresponding to the BVP (1), with homogeneous condition at $z=0$, can be derived following the method presented in $[11,13]$. Here we skip the details and we simply present the final expression: 


$$
\begin{aligned}
& G(\mathbf{x} ; \xi)=\sum_{m=0}^{\infty} \epsilon_{m} G_{m}(r, z ; \rho, \zeta) \cos m(\theta-\vartheta) \\
& G_{m}(r, z ; \rho, \zeta)=-\frac{i}{2} \sum_{n=-2}^{\infty} \mathcal{C}_{n}\left(\begin{array}{c}
H_{m}\left(\mu_{n} r\right) J_{m}\left(\mu_{n} \rho\right) \\
J_{m}\left(\mu_{n} r\right) H_{m}\left(\mu_{n} \rho\right)
\end{array}\right) f_{n}\left(\mu_{n} z\right) f_{n}\left(\mu_{n} \zeta\right), \quad\left(\begin{array}{l}
r>\rho \\
r<\rho
\end{array}\right)
\end{aligned}
$$

This expression is valid for two arbitrary points $\mathbf{x}=(r, \theta, z)$ and $\xi=(\rho, \vartheta, \zeta)$ in the fluid $-H \leq(z, \zeta) \leq 0$. For the sake of clarity, the total potential $\varphi$ is further decomposed in two parts $\varphi=\varphi_{B}+\varphi_{Q}$ by defining the following BVP's:

$$
\begin{array}{llll}
\Delta \varphi_{B}=0 & \Delta \varphi_{Q}=0 & r>a,-H<z<0 \\
-\alpha \varphi_{B}+\kappa \frac{\partial \varphi_{B}}{\partial z}=0 & -\alpha \varphi_{Q}+\kappa \frac{\partial \varphi_{Q}}{\partial z}=Q(r, \theta) & z=0 \\
\frac{\partial \varphi_{B}}{\partial n}=v(a, z, \theta) & \frac{\partial \varphi_{Q}}{\partial n}=0 & r=a \\
\frac{\partial \varphi_{B}}{\partial z}=0 & \frac{\partial \varphi_{Q}}{\partial z}=0 & z=-H \\
\varphi_{B} \rightarrow 0 & \varphi_{Q} \rightarrow 0 & r \rightarrow \infty
\end{array}
$$

In addition it should be noted that both potentials should also satisfy the conditions at the plate ends (3). The potential $\varphi_{B}$ is called body perturbation potential and it represents the generic linear (first order) potential with homogeneous condition at the free surface. The potential $\varphi_{Q}$ is called free surface perturbation potential and it satisfy the nonhomogeneous condition at the free surface together with the homogeeous condition at the cylinder. This potential is relevant for higher order problems $[2,3,15,16]$.

\subsection{Potential $\varphi_{B}$}

The solution for the body potential $\varphi_{B}$ will be obtained using two different methods namely: the direct eigenfunctions expansion method and the Boundary Integral Equation method. Both methods are described in details below.

\subsubsection{Direct eigenfunctions expansion method}

Since the potential $\varphi_{B}$ satisfies the homogeneous condition at the free surface, it can be shown that the following eigenfunction expansion can describe any particular wave system generated by the bottom mounted vertical circular cylinder:

$$
\varphi_{B}(r, \theta, z)=\sum_{m=0}^{\infty} \epsilon_{m} \varphi_{B m}(r, z) \cos m \theta=\sum_{m=0}^{\infty} \epsilon_{m} \sum_{n=-2}^{\infty} f_{n}(z) \beta_{m n} H_{m}\left(\mu_{n} r\right) \cos m \theta
$$

where $\beta_{m n}$ are the unknown coefficients and $H_{m}$ denotes the Hankel function of the first kind. 
It is important to note that the above expression (17) is written in compact form which means, in particular, that for $n \geq 1$ we have:

$$
\begin{aligned}
& f_{n}(z)=\frac{\cosh i \mu_{n}(z+H)}{\cosh i \mu_{n} H}=\frac{\cos k_{n}(z+H)}{\cos k_{n} H}, \\
& K_{m}\left(k_{n} r\right)=\frac{\pi}{2} i^{m+1} H_{m}\left(i \mu_{n} r\right) \quad, \quad I_{m}\left(k_{n} r\right)=i^{-m} J_{m}\left(i \mu_{n} r\right)
\end{aligned}
$$

We now apply the body boundary condition (16) to $\varphi_{B}$ and we get:

$$
\sum_{n=-2}^{\infty} f_{n}(z) \beta_{m n} \mu_{n} H_{m}^{\prime}\left(\mu_{n} a\right)=v_{m}(a, z)
$$

where $v_{m}(a, z)$ are the Fourier components of the body velocity.

After multiplying the above equation by $f_{k}(z)$ and integrating over the depth, we obtain:

$$
\sum_{n=-2}^{\infty} \beta_{m n} \mu_{n} H_{m}^{\prime}\left(\mu_{n} a\right) \int_{-H}^{0} f_{k}(z) f_{n}(z) d z=\int_{-H}^{0} f_{k}(z) v_{m}(a, z) d z
$$

By using the orthogonality properties (13) this can be rewritten in the form:

$$
\sum_{n=-2}^{\infty} \beta_{m n} \mu_{n} H_{m}^{\prime}\left(\mu_{n} a\right)\left[\frac{\delta_{k n}}{2 \mathcal{C}_{n}}-\frac{\mathcal{D}}{\alpha}\left(f_{k}^{\prime \prime \prime} f_{n}^{\prime}+f_{k}^{\prime} f_{n}^{\prime \prime \prime}\right)_{z=0}\right]=\int_{-H}^{0} f_{k}(z) v_{m}(a, z) d z
$$

which is the same as:

$$
\frac{1}{2 \mathcal{C}_{k}} \beta_{m k} \mu_{k} H_{m}^{\prime}\left(\mu_{k} a\right)-\frac{\mathcal{D}}{\alpha}\left[f_{k}^{\prime \prime \prime} \frac{\partial^{2} \varphi_{B m}}{\partial r \partial z}+f_{k}^{\prime} \frac{\partial^{4} \varphi_{B m}}{\partial r \partial z^{3}}\right]_{\substack{r=a \\ z=0}}=\int_{-H}^{0} f_{k}(z) v_{m}(a, z) d z
$$

Knowing that $f_{k}^{\prime}=-\mu_{k} \tanh \mu_{k} H$ and $f_{k}^{\prime \prime \prime}=\mu_{k}^{3} \tanh \mu_{k} H$ we can write the unknown coefficients $\beta_{m k}$ in the following form:

$$
\beta_{m k}=\frac{2 \mathcal{C}_{k}}{\mu_{k} H_{m}^{\prime}\left(\mu_{k} a\right)}\left[\int_{-H}^{0} f_{k}(z) v_{m}(a, z) d z+\mu_{k} \tanh \mu_{k} H \frac{\mathcal{D}}{\alpha}\left(\mu_{k}^{2} \frac{\partial^{2} \varphi_{B m}}{\partial r \partial z}-\frac{\partial^{4} \varphi_{B m}}{\partial r \partial z^{3}}\right)_{\substack{r=a \\ z=0}}\right]
$$

The values of $\partial^{2} \varphi_{B m} / \partial r \partial z$ and $\partial^{4} \varphi_{B m} / \partial r \partial z^{3}$, at the connecting line $(a, 0)$, are unknown in advance and can be found from the appropriate edge conditions. In order to simplify the notations, we introduce the following notations:

$$
\gamma_{m}=\frac{\partial^{2} \varphi_{B m}}{\partial r \partial z}(a, 0) \quad, \quad \sigma_{m}=\frac{\partial^{4} \varphi_{B m}}{\partial r \partial z^{3}}(a, 0)
$$

When applying the edge conditions, and whatever the type of these conditions, the system of equations for $\gamma_{m}$ and $\sigma_{m}$ can be deduced in the following form: 


$$
\begin{aligned}
& J_{1} \gamma_{m}+J_{2} \sigma_{m}=J_{3} \\
& J_{4} \gamma_{m}+J_{5} \sigma_{m}=J_{6}
\end{aligned}
$$

where $J_{n}, n=1,6$ are the known coefficients [6].

Solution of this system gives the coefficients $\gamma_{m}$ and $\sigma_{m}$ so that the problem is formally solved.

\subsubsection{Boundary Integral Equation method}

After applying the Green's theorem to the unknown velocity potential $\varphi_{B}(\mathbf{x})$ and the Green's function $G(\mathbf{x} ; \xi)$, the following Boundary Integral Equation can be written:

$$
\begin{array}{r}
\left(\begin{array}{l}
4 \pi \varphi_{B}(\mathbf{x}) \\
0
\end{array}\right)-\iint_{S_{a}} \varphi_{B}(\xi) \frac{\partial G(\mathbf{x} ; \xi)}{\partial \rho} d S=\iint_{S_{a}} G(\mathbf{x} ; \xi) \frac{\partial \varphi_{B}(\xi)}{\partial \rho} d S+ \\
\iiint_{S_{F}}\left[\varphi_{B}(\xi) \frac{\partial G(\mathbf{x} ; \xi)}{\partial \zeta}-G(\mathbf{x} ; \xi) \frac{\partial \varphi_{B}(\xi)}{\partial \zeta}\right] d S, \quad\left(\begin{array}{l}
r>a \\
r<a
\end{array}\right)
\end{array}
$$

where $S_{a}$ denotes the surface of the cylinder $r=a, S_{F}$ denotes the free surface $z=0$, and where it was accounted for the fact that the integrals at infinity and at the sea bottom ( $z=-H$ ) disappear.

For convenience we denote the free surface integral in (28) by $I_{S_{F}}$ and we rewrite it as follows:

$$
\begin{aligned}
I_{S_{F}} & =\iint_{S_{F}}\left[\varphi_{B} \frac{\partial G}{\partial \zeta}-G \frac{\partial \varphi_{B}}{\partial \zeta}\right] d S= \\
& =\frac{1}{\alpha} \iint_{S_{F}}\left[\left(\kappa \frac{\partial \varphi_{D}}{\partial \zeta}\right) \frac{\partial G}{\partial \zeta}-\left(\kappa \frac{\partial G}{\partial \zeta}\right) \frac{\partial \varphi_{D}}{\partial \zeta}\right] d S=\frac{\mathcal{D}}{\alpha} \iint_{S_{F}}\left[\frac{\partial G}{\partial \zeta} \Delta_{0}^{2} \frac{\partial \varphi_{B}}{\partial \zeta}-\frac{\partial \varphi_{B}}{\partial \zeta} \Delta_{0}^{2} \frac{\partial G}{\partial \zeta}\right] d S
\end{aligned}
$$

Now we use the following identity which is valid for two arbitrary harmonic functions $\phi$ and $\psi$ :

$$
\iint_{S}\left(\psi \Delta^{2} \phi-\phi \Delta^{2} \psi\right) d S=\int_{C}\left[\Delta \psi \frac{\partial \phi}{\partial n}-\Delta \phi \frac{\partial \psi}{\partial n}+\psi \frac{\partial}{\partial n} \Delta \phi-\phi \frac{\partial}{\partial n} \Delta \psi\right] d C
$$

where $S$ denotes the closed surface and $C$ its contour(s).

This allows rewriting the integral $I_{S_{F}}$ in the following form:

$$
\begin{aligned}
I_{S_{F}} & =\frac{\mathcal{D}}{\alpha} \int_{r=a}\left[\Delta_{0} \frac{\partial G}{\partial \zeta} \frac{\partial^{2} \varphi_{B}}{\partial n \partial \zeta}-\Delta_{0} \frac{\partial \varphi_{B}}{\partial \zeta} \frac{\partial^{2} G}{\partial n \partial \zeta}+\frac{\partial G}{\partial \zeta} \frac{\partial}{\partial n}\left(\Delta_{0} \frac{\partial \varphi_{B}}{\partial \zeta}\right)-\frac{\partial \varphi_{B}}{\partial \zeta} \frac{\partial}{\partial n}\left(\Delta_{0} \frac{\partial G}{\partial \zeta}\right)\right] d C \\
& =-\frac{\mathcal{D}}{\alpha} \int_{r=a}\left[\frac{\partial^{3} G}{\partial \zeta^{3}} \frac{\partial^{2} \varphi_{B}}{\partial \rho \partial \zeta}-\frac{\partial^{3} \varphi_{B}}{\partial \zeta^{3}} \frac{\partial^{2} G}{\partial \rho \partial \zeta}+\frac{\partial G}{\partial \zeta} \frac{\partial^{4} \varphi_{B}}{\partial \rho \partial \zeta^{3}}-\frac{\partial \varphi_{B}}{\partial \zeta} \frac{\partial^{4} G}{\partial \rho \partial \zeta^{3}}\right] d C
\end{aligned}
$$


After exploiting the orthogonality properties of the Fourier series, we can write for one point inside the cylinder $[r=a-\delta(\delta>0)]$, the following expression:

$$
\begin{aligned}
-\int_{H}^{0} \varphi_{B m}(a, \zeta) & \frac{\partial G_{m}(r, z ; a, \zeta)}{\partial \rho} d \zeta=\int_{-H}^{0} G_{m}(r, z ; a, \zeta) v_{m}(a, \zeta) d \zeta \\
-\frac{\mathcal{D}}{\alpha} & {\left[\frac{\partial^{3} G_{m}}{\partial \zeta^{3}} \frac{\partial^{2} \varphi_{B m}}{\partial \rho \partial \zeta}-\frac{\partial^{3} \varphi_{B m}}{\partial \zeta^{3}} \frac{\partial^{2} G_{m}}{\partial \rho \partial \zeta}+\frac{\partial G_{m}}{\partial \zeta} \frac{\partial^{4} \varphi_{B m}}{\partial \rho \partial \zeta^{3}}-\frac{\partial \varphi_{B m}}{\partial \zeta} \frac{\partial^{4} G_{m}}{\partial \rho \partial \zeta^{3}}\right]_{\substack{\rho=a \\
\zeta=0}} }
\end{aligned}
$$

For the sake of clarity, we rewrite the above equation in compact form:

$$
-\mathcal{L}^{B}=\mathcal{P}^{B}+\frac{\mathcal{D}}{\alpha}\left(\mathcal{Q}_{1}^{B}-\mathcal{Q}_{2}^{B}\right)
$$

and we explicit below the different terms:

$$
\begin{aligned}
& \mathcal{L}^{B}=\int_{-H}^{0} \varphi_{B m}(a, \zeta) \frac{\partial G_{m}(r, z ; a, \zeta)}{\partial \rho} d \zeta \\
& =-\sum_{k=-2}^{\infty} \beta_{m k} H_{m}\left(\mu_{k} a\right) \frac{i}{2} \mathcal{C}_{k} J_{m}\left(\mu_{k} r\right) \mu_{k} H_{m}^{\prime}\left(\mu_{k} a\right) f_{k}\left(\mu_{k} z\right) \frac{1}{2 \mathcal{C}_{k}}-\frac{\mathcal{D}}{\alpha} \mathcal{Q}_{1}^{B} \\
& \mathcal{P}^{B}=\int_{-H}^{0} G_{m}(r, z ; a, \zeta) v_{m}(a, \zeta) d \zeta \\
& =-\sum_{k=-2}^{\infty} \frac{i}{2} \mathcal{C}_{k} J_{m}\left(\mu_{k} r\right) H_{m}\left(\mu_{k} a\right) f_{k}\left(\mu_{k} z\right) \int_{-H}^{0} f_{k}\left(\mu_{k} \zeta\right) v_{m}(a, \zeta) d \zeta \\
& \mathcal{Q}_{1}^{B}=\left[\frac{\partial^{3} \varphi_{B m}}{\partial \zeta^{3}} \frac{\partial^{2} G_{m}}{\partial \rho \partial \zeta}+\frac{\partial \varphi_{B m}}{\partial \zeta} \frac{\partial^{4} G_{m}}{\partial \rho \partial \zeta^{3}}\right]_{\substack{\rho=a \\
\zeta=0}} \\
& =\sum_{n=-2}^{\infty} \beta_{m n} H_{m}\left(\mu_{n} a\right)\left[f_{n}^{\prime \prime \prime} \frac{\partial^{2} G_{m}}{\partial \rho \partial \zeta}+f_{n}^{\prime} \frac{\partial^{4} G_{m}}{\partial \rho \partial \zeta^{3}}\right]_{\substack{\rho=a \\
\zeta=0}} \\
& \mathcal{Q}_{2}^{B}=\left[\frac{\partial^{3} G_{m}}{\partial \zeta^{3}} \frac{\partial^{2} \varphi_{B m}}{\partial \rho \partial \zeta}+\frac{\partial G_{m}}{\partial \zeta} \frac{\partial^{4} \varphi_{B m}}{\partial \rho \partial \zeta^{3}}\right]_{\substack{\rho=a \\
\zeta=0}} \\
& =-\sum_{n=-2}^{\infty} \frac{i}{2} \mathcal{C}_{n} J_{m}\left(\mu_{n} r\right) H_{m}\left(\mu_{n} a\right) f_{n}\left(\mu_{n} z\right)\left[f_{n}^{\prime \prime \prime} \frac{\partial^{2} \varphi_{B m}}{\partial \rho \partial \zeta}+f_{n}^{\prime} \frac{\partial^{4} \varphi_{B m}}{\partial \rho \partial \zeta^{3}}\right]_{\substack{\rho=a \\
\zeta=0}}
\end{aligned}
$$

With this in mind the equation (32) becomes:

$$
\begin{aligned}
\sum_{k=-2}^{\infty}-\frac{i}{2} \mathcal{C}_{k} J_{m}\left(\mu_{k} r\right) H_{m}\left(\mu_{k} a\right) f_{k}\left(\mu_{k} z\right) \cdot & \\
& {\left[\frac{1}{2 \mathcal{C}_{k}} \beta_{m k} \mu_{k} H_{m}^{\prime}\left(\mu_{k} a\right)-\int_{-H}^{0} f_{k}\left(\mu_{k} \zeta\right) v_{m}(a, \zeta) d \zeta-\frac{\mathcal{D}}{\alpha}\left(f_{k}^{\prime \prime \prime} \frac{\partial^{2} \varphi_{B m}}{\partial \rho \partial \zeta}+f_{k}^{\prime} \frac{\partial^{4} \varphi_{B m}}{\partial \rho \partial \zeta^{3}}\right)_{\substack{\rho=a \\
\zeta=0}}\right]=0 }
\end{aligned}
$$


Since the above equation should be valid for any $r$ and any $z$ inside the cylinder, the only solution is that the term under the brackets is equal to zero. This leads exactly to the equation (23) which proves that the two solutions are identical.

\section{Potential $\varphi_{Q}$}

Due to the fact that the potential $\varphi_{Q}$ satisfies the non homogeneous condition at the free surface, it is not possible to derive the explicit dependence of the velocity potential in radial direction. However, at each radial distance the eigenfunction expansions in vertical and circumferental directions can be applied. In that respect we can write for the velocity potential at the cylinder surface $(r=a)$ :

$$
\varphi_{Q}(a, \theta, z)=\sum_{m=0}^{\infty} \epsilon_{m} \varphi_{Q m}(a, z) \cos m \theta=\sum_{m=0}^{\infty} \epsilon_{m} \sum_{n=-2}^{\infty} f_{n}(z) A_{m n} \cos m \theta
$$

The solution for this potential will be obtained using the boundary integral equation method only. In this case, the Green's theorem for a point inside the cylinder, gives:

$$
\iint_{S_{a}} \varphi_{Q}(\xi) \frac{\partial G(\mathbf{x} ; \xi)}{\partial \rho} d S=I_{S_{F}}
$$

where $I_{S_{F}}$ is now:

$$
\begin{aligned}
I_{S_{F}} & =\iint_{S_{F}}\left[\varphi_{Q} \frac{\partial G}{\partial \zeta}-G \frac{\partial \varphi_{Q}}{\partial \zeta}\right] d S=\frac{1}{\alpha} \iint_{S_{w}}\left[\left(\kappa \frac{\partial \varphi_{Q}}{\partial \zeta}\right) \frac{\partial G}{\partial \zeta}-\left(\kappa \frac{\partial G}{\partial \zeta}\right) \frac{\partial \varphi_{Q}}{\partial \zeta}\right] d S \\
& =\frac{1}{\alpha} \iint_{S_{F}} \frac{\partial G}{\partial \zeta} Q d S+\frac{\mathcal{D}}{\alpha} \iint_{S_{F}}\left[\frac{\partial G}{\partial \zeta} \Delta_{0}^{2} \frac{\partial \varphi_{Q}}{\partial \zeta}-\frac{\partial \varphi_{Q}}{\partial \zeta} \Delta_{0}^{2} \frac{\partial G}{\partial \zeta}\right] d S
\end{aligned}
$$

After using the orthogonality of the Fourier series we can write for a point inside the cylinder the following expression:

$$
\begin{aligned}
\int_{-H}^{0} \varphi_{Q m}(a, \zeta) & \frac{\partial G_{m}(r, z ; a, \zeta)}{\partial \rho} d \zeta=-\frac{1}{a \alpha} \int_{a}^{\infty} \frac{\partial G_{m}(r, z ; \rho, 0)}{\partial \zeta} Q_{m}(\varrho) d \rho \\
+ & \frac{\mathcal{D}}{\alpha}\left[\frac{\partial^{3} G_{m}}{\partial \zeta^{3}} \frac{\partial^{2} \varphi_{Q m}}{\partial \rho \partial \zeta}-\frac{\partial^{3} \varphi_{Q m}}{\partial \zeta^{3}} \frac{\partial^{2} G_{m}}{\partial \rho \partial \zeta}+\frac{\partial G_{m}}{\partial \zeta} \frac{\partial^{4} \varphi_{Q m}}{\partial \rho \partial \zeta^{3}}-\frac{\partial \varphi_{Q m}}{\partial \zeta} \frac{\partial^{4} G_{m}}{\partial \rho \partial \zeta^{3}}\right]_{\substack{\rho=a \\
\zeta=0}}
\end{aligned}
$$

Similar to body perturbation potential, we rewrite the above expression in compact form as follows:

$$
\mathcal{L}^{Q}=-\frac{1}{a \alpha} \mathcal{P}^{Q}+\frac{\mathcal{D}}{\alpha}\left(\mathcal{Q}_{1}^{Q}-\mathcal{Q}_{2}^{Q}\right)
$$

where the different terms can be deduced in the following form:

$$
\begin{aligned}
& \mathcal{L}^{Q}=\sum_{k=-2}^{\infty} A_{m k} \frac{i}{2} \mathcal{C}_{k} J_{m}\left(\mu_{k} r\right) \mu_{k} a H_{m}^{\prime}\left(\mu_{k} a\right) f_{k}\left(\mu_{k} z\right) \frac{1}{2 \mathcal{C}_{k}}-\frac{\mathcal{D}}{\alpha} \mathcal{Q}_{1}^{Q} \\
& \mathcal{P}^{Q}=\int_{a}^{\infty} \frac{\partial G_{m}(r, z ; \rho, 0)}{\partial \zeta} Q_{m}(\varrho) d \rho
\end{aligned}
$$




$$
\begin{aligned}
& =\sum_{k=-2}^{\infty} \frac{i}{2} \mathcal{C}_{k} J_{m}\left(\mu_{k} r\right) f_{k}\left(\mu_{k} z\right) \mu_{k} \tanh \mu_{k} H \int_{a}^{\infty} H_{m}\left(\mu_{k} \rho\right) Q_{m}(\varrho) d \rho \\
\mathcal{Q}_{1}^{Q}= & \sum_{n=-2}^{\infty} A_{m n}\left[f_{n}^{\prime \prime \prime} \frac{\partial^{2} G_{m}}{\partial \rho \partial \zeta}+f_{n}^{\prime} \frac{\partial^{4} G_{m}}{\partial \rho \partial \zeta^{3}}\right]_{\substack{\rho=a \\
\zeta=0}} \\
\mathcal{Q}_{2}^{Q}= & -\sum_{n=-2}^{\infty} \frac{i}{2} \mathcal{C}_{n} J_{m}\left(\mu_{n} r\right) H_{m}\left(\mu_{n} a\right) f_{n}\left(\mu_{n} z\right)\left[f_{n}^{\prime \prime \prime} \frac{\partial^{2} \varphi_{B m}}{\partial \rho \partial \zeta}+f_{n}^{\prime} \frac{\partial^{4} \varphi_{B m}}{\partial \rho \partial \zeta^{3}}\right]_{\substack{\rho=a \\
\zeta=0}}
\end{aligned}
$$

After collecting all the different terms we can rewrite the equation (42) as below:

$$
\begin{aligned}
& \sum_{k=-2}^{\infty} \frac{i}{2} \mathcal{C}_{k} J_{m}\left(\mu_{k} r\right) f_{k}\left(\mu_{k} z\right) \cdot\left[\frac{1}{2 \mathcal{C}_{k}} A_{m k} \mu_{k} a H_{m}^{\prime}\left(\mu_{k} a\right)\right. \\
& \left.\quad-\frac{\mu_{k} \tanh \mu_{k} H}{\alpha} \int_{a}^{\infty} H_{m}\left(\mu_{k} \rho\right) Q_{m}(\varrho) d \rho-a H_{m}\left(\mu_{k} a\right) \frac{\mathcal{D}}{\alpha}\left(f_{k}^{\prime \prime \prime} \frac{\partial^{2} \varphi_{Q m}}{\partial \rho \partial \zeta}+f_{k}^{\prime} \frac{\partial^{4} \varphi_{Q m}}{\partial \rho \partial \zeta^{3}}\right)_{\substack{\rho=a \\
\zeta=0}}\right]=0
\end{aligned}
$$

Since this equation should be valid for any $r$ and any $z$ inside the cylinder, the only solution is that the term under the brackets is equal to zero. Furthermore, knowing that $f_{k}^{\prime}=-\mu_{k} \tanh \mu_{k} H$ and $f_{k}^{\prime \prime \prime}=\mu_{k}^{3} \tanh \mu_{k} H$ we can write the unknown coefficients $A_{m k}$ in the following form:

$$
\begin{aligned}
A_{m k}= & \frac{-2 \mathcal{C}_{k}}{\mu_{k} a H_{m}^{\prime}\left(\mu_{k} a\right)} \frac{\mu_{k} \tanh \mu_{k} H}{\alpha} . \\
& {\left[\int_{a}^{\infty} H_{m}\left(\mu_{k} \rho\right) Q_{m}(\varrho) d \rho-a \mathcal{D} H_{m}\left(\mu_{k} a\right)\left(\mu_{k}^{2} \frac{\partial^{2} \varphi_{Q m}}{\partial r \partial z}-\frac{\partial^{4} \varphi_{Q m}}{\partial r \partial z^{3}}\right)_{\substack{r=a \\
z=0}}\right] }
\end{aligned}
$$

The quantities $\partial^{2} \varphi_{Q m} / \partial r \partial z$ and $\partial^{4} \varphi_{Q m} / \partial r \partial z^{3}$, are evaluated in the same way as in the first order case i.e. through the application of the edge conditions.

\section{Some particular cases}

The above described procedure is now applied to some particular wave body interaction problems. In order to demonstrate the generality of the proposed approaches, we first apply the methods to the case of the water wave interactions and we show that the classical results are recovered. After that the case of the linear wave diffraction is considered and it is shown that the classical solution from [1] is recovered at the end.

\subsection{Water waves}

In order to demonstrate the generality of the proposed approaches, here we apply the methods to the case of the water wave interactions and we show that the classical results are easily obtained by simply applying the expressions given here. 
In the case of the water waves we have $M=0$ and $D=0$ and, at the same time, the edge conditions (3) are no more relevant. With this in mind, the classical dispersion relation for water waves is easily recovered:

$$
\alpha=\mu \tanh \mu H
$$

The solution of this equation gives one positive $\operatorname{root}\left(\mu_{0}\right)$ and an infinite number of purely imaginary roots $\left(i \mu_{n}, n=1, \infty\right)$.

The corresponding eigenfunctions in vertical direction keep the same form (11) and the orthogonality relation simplifies to:

$$
\int_{-H}^{0} f_{m}(z) f_{n}(z)=\frac{\delta_{m n}}{2 C_{n}}= \begin{cases}0 & n \neq m \\ \frac{1}{2 C_{n}} & n=m\end{cases}
$$

The unknown coefficients for the body perturbation potential become:

$$
\beta_{m n}=-\frac{2 C_{n}}{\mu_{n} H_{m}^{\prime}\left(\mu_{n} a\right)} \int_{-H}^{0} f_{n}(z) v_{m}(a, z) d z
$$

and those for the free surface perturbation potential become:

$$
A_{m n}=-\frac{2 C_{n}}{\mu_{n} a H_{m}^{\prime}\left(\mu_{n} a\right)} \int_{a}^{\infty} H_{m}\left(\mu_{n} \varrho\right) Q_{m}(\varrho) d \rho
$$

These expressions are the same as those in [3].

\subsection{Diffraction}

Only the case of the linear diffraction of monochromatic waves is considered.

\subsubsection{Flexural gravity waves}

The incident wave field is defined as the progressive sinusoidal wave with the folowing expression for the corresponding velocity potential $\varphi_{I}$ :

$$
\varphi_{I}=\mathcal{A} f_{0}(z) \sum_{m=0}^{\infty} \epsilon_{m} i^{m} J_{m}\left(\mu_{0} r\right) \cos m \theta
$$

where $\mathcal{A}=-i g \zeta_{A} / \omega$ and $\zeta_{A}$ is the wave amplitude.

The body perturbation potential is given by (17) with the unknown coefficients $\beta_{m k}$ given by (53).

Knowing that:

$$
\begin{aligned}
\int_{-H}^{0} f_{k}(z) f_{0}(z) d z & =\frac{\delta_{k 0}}{2 \mathcal{C}_{0}}+\frac{\mathcal{D}}{\alpha}\left(f_{k}^{\prime \prime \prime} f_{0}^{\prime}+f_{k}^{\prime} f_{0}^{\prime \prime \prime}\right)_{z=0} \\
& =\frac{\delta_{k 0}}{2 \mathcal{C}_{0}}-\mu_{k} \mu_{0} \tanh \mu_{k} H \tanh \mu_{0} H \frac{\mathcal{D}}{\alpha}\left(\mu_{k}^{2}+\mu_{0}^{2}\right)
\end{aligned}
$$

we can easily deduce the following expression for $\beta_{m k}$ : 


$$
\begin{aligned}
\beta_{m k} & =-\mathcal{A} i^{m} \frac{J_{m}^{\prime}\left(\mu_{0} a\right)}{H_{m}^{\prime}\left(\mu_{0} a\right)} \delta_{k 0} \\
& +\frac{2 \mathcal{C}_{k} \tanh \mu_{k} H}{H_{m}^{\prime}\left(\mu_{k} a\right)} \frac{\mathcal{D}}{\alpha}\left[\mathcal{A} i^{m} \mu_{0} J_{m}^{\prime}\left(\mu_{0} a\right) \tanh \mu_{0} H\left(\mu_{k}^{2}+\mu_{0}^{2}\right)+\left(\mu_{k}^{2} \gamma_{m}-\sigma_{m}\right)\right]
\end{aligned}
$$

or:

$$
\begin{aligned}
& \beta_{m 0}=-\mathcal{A} i^{m} \frac{J_{m}^{\prime}\left(\mu_{0} a\right)}{H_{m}^{\prime}\left(\mu_{0} a\right)}+\frac{2 \mathcal{C}_{0} \tanh \mu_{0} H}{H_{m}^{\prime}\left(\mu_{0} a\right)} \frac{\mathcal{D}}{\alpha}\left[2 \mathcal{A} i^{m} \mu_{0}^{3} J_{m}^{\prime}\left(\mu_{0} a\right) \tanh \mu_{0} H+\left(\mu_{0}^{2} \gamma_{m}-\sigma_{m}\right)\right] \\
& \beta_{m k}=\frac{2 \mathcal{C}_{k} \tanh \mu_{k} H}{H_{m}^{\prime}\left(\mu_{k} a\right)} \frac{\mathcal{D}}{\alpha}\left[\mathcal{A} i^{m} \mu_{0} J_{m}^{\prime}\left(\mu_{0} a\right) \tanh \mu_{0} H\left(\mu_{k}^{2}+\mu_{0}^{2}\right)+\left(\mu_{k}^{2} \gamma_{m}-\sigma_{m}\right)\right]
\end{aligned}
$$

where $k \neq 0$.

It can be shown that this solution is identical to the solution proposed in [8].

\subsubsection{Water waves}

In the case of water waves the well known MacCamy and Fuchs solution is recovered:

$$
\beta_{m 0}=-\mathcal{A} i^{m} \frac{J_{m}^{\prime}\left(\mu_{0} a\right)}{H_{m}^{\prime}\left(\mu_{0} a\right)}
$$

with $\beta_{m k}=0$ for $k>0$.

\section{Conclusions}

We presented here an alternative semi-analytical method for the solution of the interactions in between the flexural gravity waves and the bottom mounted vertical circular cylinder. The proposed method covers both the linear (body perturbation potential) and the higher order solution (free surface perturbation potential). The linear solution was obtained by two different methods namely direct eigenfunction expansion method and the boundary integral equation method, while the higher order solution was found using the boundary integral equation method only. For the linear case, it was shown that the final expressions are equivalent to other expressions proposed in the literature. The higher order solution for flexural gravity waves is new and was not reported before in the literature.

\section{Acknowledgments}

The support from a National Research Foundation of Korea (NRF) grant funded by the Korean Government (MSIP) through GCRC-SOP (Grant No. 2011-0030013) is acknowledged. Discussions and advices of Prof. Korobkin A.A. and Prof. Khabakhpasheva T. are also greatly appreciated.

\section{REFERENCES}

[1] Mac Camy R.C. \& Fuchs R.A. 1954. : Wave forcs on piles: A diffraction theory., Report No. 69, U.S. Army Corps of Engineers, Beach Erosion Board, Washington D.C.

[2] Chau F.P. \& Eatock Taylor R. 1992. : Second order wave diffraction by a vertical cylinder, J.Fluid Mech., Vol. 240, pp. 571-599 https://doi.org/10.1017/S0022112092000211 
[3] Malenica S. \& Molin B. 1995. : "Third-harmonic wave diffraction by a vertical cylinder.", Journal of Fluid Mech., vol. 302, pp.203-229 https://doi.org/10.1017/S0022112095004071

[4] Evans D.V. \& Meylan M.H. 2005. : "Scattering of flexural waves by a pinned thin elastic sheet floating on water.", 20th IWWWFB, Longyearbyen, Norway

[5] Fox C. \& Chung H. 2002. :"Harmonic deflections of an infinite floating plate.", University of Auckland, Dept. of Mathematics Report

[6] Bhattacharjee J. \& Guedes Soares C. 2012. : Flexural gravity wave over a floating ice sheet near a vertical wall., J.Eng. Math., Vol. 75, pp. 29-48. https://doi.org/10.1007/s10665-011-9511-3

[7] Manam S.R. \& Bhattacharjee J. \& Sahoo T. 2006. : Expansion formulae in wave structure interaction problems., Proc. of R. Soc. A, Vol. 462, pp. 263-287. https://doi.org/10.1098/rspa.2005.1562

[8] Korobkin A.A., Malenica S. \& Khabakhpasheva T.I. 2018. : Interaction of flexural gravity waves in ice cover with vertical walls., Phil. Trans. R. Soc. A, 376(21219), 20170347. https://doi.org/10.1098/rsta.2017.0347

[9] Korobkin A.A., Khabakhpasheva T.I. \& Papin A.A. 2014. : Waves propagating along a channel with ice cover., European Journal of Mechanics - B,/ Fluids, 166-175. https://doi.org/10.1016/j.euromechflu.2014.01.007

[10] Shishmarev K., Khabakhpasheva T.I., \& Korobkin A.A. 2016. : The response of ice cover to a load moving along a frozen channel., Applied Ocean Research, 59, 313-326. https://doi.org/10.1016/j.apor.2016.06.008

[11] Mandal S., Sahoo T. \& Chakrabarti A. 2015. : ‘ A note onconvergence of expansion formulae for wave structure interaction problems., 30th IWWWFB, Bristol, UK

[12] Malenica S. \& Korobkin A.A. 2003. : "Water wave diffraction by vertical circular cylinder in partially frozen sea.", 18th IWWWFB, Nantes, France

[13] Evans D. \& Porter R. 2003. : Wave scattering by narrow cracks in ice sheets floating on water of finite depth., J.Fluid Mech., Vol. 484, pp. 143-165. https://doi.org/10.1017/S002211200300435X

[14] Ren K., Wu G.X. \& Ji C.Y. 2018. : Diffraction of hydroelastic waves by multiple vertical circular cylinders., J. Engg. Math., doi. 10.1007/s10665-018-9973-7

[15] Hegarty G.M. \& Squire V.A. 2008. : A boundary integral method for the interaction of large amplitude ocean waves with a compliant floating raft such as sea ice flo., J. Engg. Math., doi. 10.1007/s10665-0089219-1

[16] Han S.Y., Malenica S., Kim Y.J., Kim B.J. \& Kwon S.H. 2015. : Second order hydroelastic response of floating circular plate. Part 1: Theory., 7th Int. Conf. on Hydroelasticity in Marine Tech., Split, Croatia

Submitted: $\quad$ 07.12.2018. Šime Malenica, sime.malenica@bureauveritas.com

Accepted: $\quad$ 15.01.2019. Paris, FRANCE 